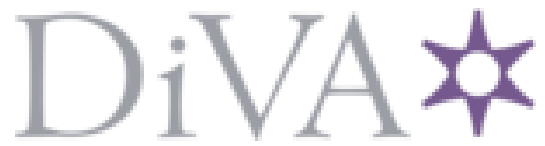

http://www.diva-portal.org

Postprint

This is the accepted version of a paper presented at 36th IEEE Global Communications Conference (GLOBECOM), DEC 04-O8, 2017, Singapore, SINGAPORE.

Citation for the original published paper:

Hoang, L-N., Uhlemann, E., Jonsson, M. (2017)

Relaying with Packet Aggregation for Half-Duplex All-to-All Broadcast in Time-Critical Wireless Networks

In: 2017 IEEE GLOBECOM WORKSHOPS (GC WKSHPS) IEEE

IEEE Globecom Workshops

https://doi.org/10.1109/GLOCOMW.2017.8269134

N.B. When citing this work, cite the original published paper.

Permanent link to this version:

http://urn.kb.se/resolve?urn=urn:nbn:se:mdh:diva-38896 


\title{
Relaying with Packet Aggregation for Half-Duplex All-to-All Broadcast in Time-Critical Wireless Networks
}

\author{
Le-Nam Hoang*, Elisabeth Uhlemann*† and Magnus Jonsson* \\ * CERES, Halmstad University, Halmstad, Sweden \\ $\dagger$ Malardalen University, Västerås, Sweden \\ Email: \{le-nam.hoang, elisabeth.uhlemann, magnus.jonsson\}@hh.se, elisabeth.uhlemann@mdh.se
}

\begin{abstract}
Wireless automation and control networks, with stringent latency and reliability requirements, typically use halfduplex communications combined with deadline-aware scheduling of time slots to nodes. To introduce higher reliability in legacy industrial control systems, extra time slots are usually reserved for retransmissions. However, in distributed wireless control systems, where sensor data from several different nodes must be timely and reliably available at all places where controller decisions are made, this is particularly cumbersome as all nodes may not hear each other and extra time slots imply increased delay. To enable all-to-all broadcast with manageable overhead and complexity in such systems, we therefore propose a novel relaying strategy using packet aggregation. The strategy assigns relayers to time slots, as well as determines which packets to aggregate in each slot, using a low-complexity algorithm such that ultra-reliable communications can be obtained with maintained end-to-end latency.
\end{abstract}

Index Terms-Broadcasting; Error Probability; Latency; Reliability.

\section{INTRODUCTION}

All-to-all broadcast, i.e. each node in the network broadcasting messages to all other nodes in the network, is a fundamental operation in wireless ad hoc networks [1]-[4]. Due to the lossy nature of wireless communications, however, a packet originating from a specific node may not reach all other nodes in one transmission. For many applications, the probability that all nodes successfully decode each message originating from each other node in the network should be above a certain threshold. This is particularly true for wireless automation and control networks, with stringent latency and reliability requirements. Hence, it is important to use intermediate nodes to assist in broadcasting such that a desired reliability threshold can be achieved. To introduce higher reliability in legacy industrial control systems, which typically use a time division multiple access (TDMA) scheme combined with deadline-aware scheduling of time slots to nodes, this is accomplished by reserving extra time slots for retransmissions or relaying.

Simple broadcast mechanisms based on flooding can be very costly in terms of bandwidth contention and collisions, a.k.a.

The research leading to these results has received funding from the Knowledge Foundation through the SIDUS project 20130086 READY and from the SafeCOP project, funded from the ECSEL Joint Undertaking under grant agreement n 692529, and from National funding. broadcast storms [5]. Moreover, using acknowledgments is not efficient and even unfeasible sometimes due to the tremendous overhead associated with all-to-all broadcasting. Therefore, combining packets at intermediate nodes by network coding [4] or packet aggregation [6] into a single output packet before forwarding can bring a lot of benefits for all-to-all broadcasting. Most notably, since the reliability can be enhanced with maintained end-to-end latency and since broadcast packets tend to be small as compared to the size of a time-slot, packet aggregation is often preferable, and also enables to use legacy decoders [7], [8]. However, combinatorial designs for all-to-all broadcasting are non-polynomial problems [3], and thus very complex to solve. In this paper, we propose a novel relaying strategy with packet aggregation to guarantee timeliness and reliability for all-to-all broadcast in small-area wireless networks. Specifically, the major contributions of this work are:

- A relaying strategy with packet aggregation for all-to-all broadcast is proposed, subject to constraint on timeliness and reliability without acknowledgment. The strategy assigns relayers to slots, as well as determines which packets to aggregate in each slot, using low-complexity algorithms.

- A theoretical analysis is derived to characterize the reliability of our scheme. Closed form-expressions are used to select relayers and packets to aggregate, using both exhaustive search, for small examples, as well as lower complexity memory-based heuristics and greedy search.

- We conduct simulations to assess the performance of the theoretical analysis of the proposed scheme under various settings and environments.

The resulting approach is suitable for wireless distributed control systems, where sensor data from several different nodes must be timely and reliably available at all places where controller decisions are made.

\section{SYSTEM MODEL}

We consider a wireless network containing $N$ nodes in a small area in which each node needs to send information to all other nodes as shown in Fig. 1. For predictable and collisionfree channel access, we use TDMA in a periodic super-frame structure. Each time-slot is allocated to a single node as all 


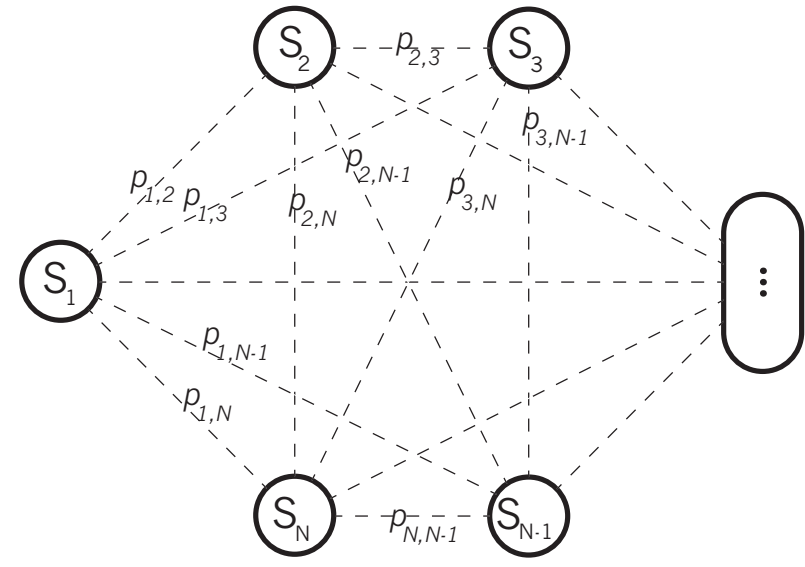

Fig. 1. An intermittent fully connected network consisting of $N$ nodes.

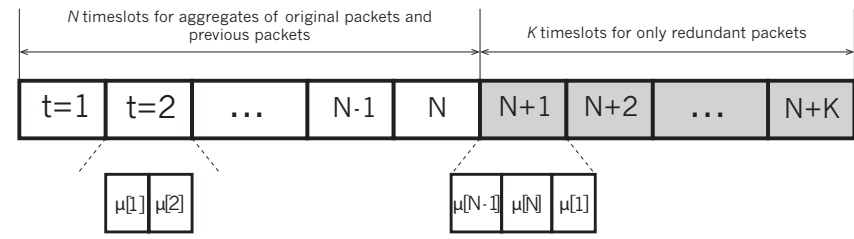

Fig. 2. The allocation of time-slots for broadcast in a super-frame in which time-slot $N+1$ conveys a big packet aggregated from $L=3$ smaller packets.

operations are in half-duplex mode, i.e. a node cannot transmit and receive simultaneously.

The channel coefficient $g_{i j}$ between any pair of nodes $(i, j)$ is an independent random variable (RV) but not identical distributed (i.n.i.d.) and characterized by its probability density function $f_{g_{i j}}\left(g_{i j}\right)$. We assume that the channel gains are stationary for the whole time-slot but can be varied in the next time-slot. Every node uses a power level $P_{t}$ to send messages, and the noise power level at the received nodes is denoted as $N_{0}$. With this setting, the probability that a message sent directly from node $i$ to node $j$ cannot be successfully decoded by node $j$ is [9],

$$
p_{i j}=\operatorname{Pr}\left[\frac{P_{t}\left|g_{i j}\right|^{2}}{N_{0}} \leq \theta_{t h}\right]=\int_{0}^{\theta_{t h}} \frac{f_{g_{i j}}\left(\sqrt{\frac{\gamma_{i j} N_{0}}{P_{t}}}\right)}{2 \sqrt{\frac{\gamma_{i j} P_{t}}{N_{0}}}} d \gamma_{i j},
$$

by denoting $\gamma_{i j}=\frac{P_{t}\left|g_{i j}\right|^{2}}{N_{0}}$. Here $\theta_{t h}$ denotes the correct decoding threshold. Note that this notation is pessimistic and assumes that if an aggregate packet is undecodable, all individual packets included in that aggregated packet are also undecodable. Further, as broadcast packets tend to be small as compared to the size of a time-slot, we assume that aggregating packets and thereby increasing the packet length has a negligible effect on the packet error rate.

\section{Relaying With Packet Aggregation}

We assume that there are totally $N+K$ time-slots allocated for all-to-all broadcasting in a super-frame as shown in Fig. 2. The first $N$ time-slots are allocated to the $N$ originating nodes, which transmit their respective source packets, possibly aggregated with one or more overheard packets. The remaining $K$ slots are used to repeat packets that the nodes assigned to the slots in this phase have overheard. For simplicity, it is assumed that all broadcast packets are of the same length and thus it is possible to aggregate at most $L$ small broadcast packets into one larger packet (see Fig. 2). In other words, each time-slot can convey $L$ small packets. During the time of one super-frame, all broadcast information is encompassed in the $N$ original packets $\mu[1], \mu[2], \ldots, \mu[N]$ transmitted in the first phase of $N$ time-slots. All other packets, aggregated either in the $N$ or in the $K$ slots, are duplicate packets to improve the reliability. Note that a packet is only valid in its current super-frame and is outdated in the next super-frame. It is assumed that the order of the original packets is given by the application, and thus it cannot be changed which node that is allocated to which slot in the first $N$ time-slots. However, the decision on which node that broadcasts which packets in which of the remaining $K$ time-slots is made by our strategy before the first transmission in each super-frame. The aim is to assign broadcasters and packets to aggregate so that after $N+K$ time-slots, the probability of all nodes having received all $N$ packets is maximized.

To provide fairness between packets, the broadcaster $\beta(t) \in$ $\{1, \ldots, N\}$ in time-slot $t$ aggregates its received packets according to the following rules:

1) $L \leq t \leq N: \beta(t)$ transmits an aggregate of its own original packet $\mu[t]$ and the $L-1$ most recent packets that $\beta(t)$ has decoded correctly in the previous time-slots before $t$.

2) $N<t \leq N+K$ : $\beta(t)$ aggregates any packets among its $L$ preferable packets that $\beta(t)$ has decoded correctly. Note that for $N<t \leq N+L-1$, the broadcasters must aggregate the $L-t+N$ most recent original packets, so that after $N+L-1$ time-slots, each packet has had at least $L$ chances to be broadcasted.

3) (Optional) If any of the preferable packets cannot be decoded at a particular node, it is replaced by another random one among the remaining successfully decoded packets.

The main contribution of this paper is the selection of the set of preferable packets to be aggregated in each time-slot, as well as the assignment of repeaters in slots $N<k \leq N+K$. These are selected while aiming to maximize the probability $B_{p}$ that all nodes successfully receive all the broadcasted messages, but using a memory-based heuristic algorithm for reduced complexity. This procedure is further explained below.

Consider an example shown in Fig. 2, with $L=3$. If the broadcaster in time-slot 2 can decode the packet $\mu[1]$ transmitted in time-slot 1, it aggregates its own original packet $\mu[2]$ with a duplicate of $\mu[1]$. The broadcaster in time-slot $N+1$, however, transmits an aggregate of duplicates of $\mu[N-1], \mu[N]$ and $\mu[1]$ originating in time-slots $N-1, N$ and 1 , respectively. Here $\mu[N-1]$ and $\mu[N]$ are chosen as they are the most recent original packets and $\mu[1]$ is chosen 
according to the strategy proposed below.

Using an $N \times N$ binary matrix $\mathbf{A}(t)$ to keep track of the decoding status of all packets at all nodes in time-slot $t$. The value of each element in $\mathbf{A}(t)$ is defined as:

$a_{s, u}(t)=\left\{\begin{array}{l}1 \text { if node } s \text { has at least one correct copy of } \\ 0 \text { otherwise. } \\ \mu[u] \text { before time-slot } t,\end{array}\right.$

Furthermore, denote $\mathbf{a}_{i,:}(t)$ as the $i$-th row of $\mathbf{A}(t)$ and $\mathbf{a}_{:, j}(t)$ as the $j$-th column of $\mathbf{A}(t)$. The probability that each node in the network successfully decodes the packets originating from all the other nodes after $N+K$ time-slots, is equivalent to the probability that all elements in $\mathbf{A}(N+K)$ are equal to 1 or $\mathbf{A}(N+K)=\mathbf{1}_{N, N}$, where $\mathbf{1}_{N, N}$ denotes the $N \times N$ matrix in which every element is equal to one.

To better illustrate the whole scheme, consider an example shown in Fig. 3 where $N=4, K=2$ and $L=3$. In the first time-slot, node $S_{1}$ transmits its original packet $\mu[1]$ but only node $S_{3}$ can receive $\mu[1]$ correctly. As $S_{2}$ is not able to decode $\mu[1]$ in the first time-slot, node $S_{2}$ only transmits its own original packet $\mu[2]$ in the second time-slot, which can be decoded only by node $S_{4}$. In time-slot 2 , the third row of $\mathbf{A}(2)$, or $\mathbf{a}_{3,:}(2)$, is $[1,0,0,0]$, hence, node $S_{3}$ broadcasts in time-slot 3 a packet in which aggregates $\mu[3]$ and $\mu[1]$. In time-slot 3 , the fourth row of $\mathbf{A}(3)$, or $\mathbf{a}_{4,:}(3)$, is $[1,1,1,0]$, but as $L=3$, node $S_{4}$ broadcasts an aggregate of $\mu[2], \mu[3]$ and $\mu[4]$ in time-slot 4 , following rule 1 . At the beginning of the second phase, $S_{3}$ is chosen to be the broadcaster in timeslot 5. Although $S_{3}$ has received packets of all other nodes, it aggregates only the packets originating from $S_{2}, S_{3}$ and $S_{4}$, following rule 2. Similarly, $S_{4}$ transmits in time-slot 6 a packet aggregating $\mu[1], \mu[2]$ and $\mu[4]$. Therefore, at the end $\mathbf{A}(6)=\mathbf{1}_{N, N}$. Note that the decision to transmit packet $\mu[2]$ in time-slot 5 and $\mu[1], \mu[2]$ in time-slot 6 is made using the strategy as explained below in Section IV.

\section{Selecting Relayers and the Preferable PACKETS TO AggREgate}

In this section, we first derive the probability that each node successfully decodes all original messages $\mu[1], \mu[2], \ldots, \mu[N]$ at the end of the super-frame when rule 3 is not used. In other words, we will derive and maximize $B_{p}=\operatorname{Pr}\left\{\mathbf{A}(N+K)=\mathbf{1}_{N, N}\right\}$. Using this expression for $B_{p}$, algorithms to allocate the broadcasters and select the preferable packets to aggregate to achieve a sufficient $B_{p}$ can be proposed.

Suppose that in one super-frame, the packet $\mu[u]$ is assigned to be transmitted in $T_{u}$ preselected time-slots $t_{k}[u]$, $k=1,2, \ldots, T_{u}$. Here $t_{k}[u]$ denotes the time-slot in which packet $\mu[u]$ is broadcasted for the $k$-th time. The sequence $\left\langle\alpha_{l}[u]\right\rangle_{h \leq l \leq k}$ is an ordered list of sub-elements $a_{\beta\left(t_{k}[u]\right), u}\left(t_{k}[u]\right)$ in $\mathbf{a}_{:, u}\left(t_{k}[u]\right)$, representing the chronological status of decoding the packet $\mu[u]$ at its respective broadcasters $\left\langle\beta_{l}[u]\right\rangle_{h \leq l \leq k}$. We denote $t_{\Lambda(k)}[u]$ as the immediate previous time-slot prior to $t_{k}[u]$ when $\beta\left(t_{k}[u]\right)$ is the broadcaster of $\mu[u]$, i.e., $\beta\left(t_{k}[u]\right)=\beta\left(t_{\Lambda(k)}[u]\right) . \Lambda(k)=0$ if $t_{k}[u]$ is the first time that $\beta\left(t_{k}[u]\right)$ is used for broadcasting $\mu[u]$. As shown in [10], the probability that the event $\alpha_{k}[u]$ takes place in timeslot $t_{k}[u]$, given that all previous events of broadcasting $\mu[u]$ are known, can be categorized into the following cases:

- The broadcaster $\beta\left(t_{k}[u]\right)$ in time-slot $t_{k}[u]$ does not aggregate packet $\mu[u]$ if and only if $\beta\left(t_{k}[u]\right)$ has not received any correct copy of the packet after overhearing in the $k$ previously allocated time-slots. On the other hand, if $\beta\left(t_{k}[u]\right)$ has successfully received at least one correct copy of the packet before time-slot $t_{\Lambda(k)}[u]$, it must transmit the packet also in time-slot $t_{k}[u]$,

$$
\begin{aligned}
& \operatorname{Pr}\left[\alpha_{k}[u]=0 \mid\left\langle\alpha_{l}[u]\right\rangle_{1 \leq l \leq k-1}, \alpha_{\Lambda(k)}[u]=1\right]=0, \\
& \operatorname{Pr}\left[\alpha_{k}[u]=1 \mid\left\langle\alpha_{l}[u]\right\rangle_{1 \leq l \leq k-1}, \alpha_{\Lambda(k)}[u]=1\right]=1 .
\end{aligned}
$$

- If $\beta\left(t_{k}[u]\right)$ did not receive a correct copy of $\mu[u]$ before time-slot $t_{\Lambda(k)}[u]$, it still has opportunities by listening in time-slots $t_{\Lambda(k)+1}[u], t_{\Lambda(k)+2}[\mathrm{u}], \ldots, t_{k-1}[u]$ and thus,

$$
\begin{aligned}
\operatorname{Pr}\left[\alpha_{k}[u]\right. & \left.=0 \mid\left\langle\alpha_{l}[u]\right\rangle_{1 \leq l \leq k-1}, \alpha_{\Lambda(k)}[u]=0\right] \\
& =\operatorname{Pr}\left[\alpha_{k}[u]=0 \mid\left\langle\alpha_{l}[u]\right\rangle_{\Lambda(k)+1 \leq l \leq k-1}\right] \\
& =\prod_{l=\Lambda(k)+1}^{k-1}\left(p_{\beta\left(t_{l}[u]\right) \beta\left(t_{k}[u]\right)}\right)^{\alpha_{l}[u]}, \\
\operatorname{Pr}\left[\alpha_{k}[u]\right. & \left.=1 \mid\left\langle\alpha_{l}[u]\right\rangle_{1 \leq l \leq k-1}, \alpha_{\Lambda(k)}[u]=0\right] \\
& =\operatorname{Pr}\left[\alpha_{k}[u]=1 \mid\left\langle\alpha_{l}[u]\right\rangle_{\Lambda(k)+1 \leq l \leq k-1}\right] \\
& =1-\prod_{l=\Lambda(k)+1}^{k-1}\left(p_{\beta\left(t_{l}[u]\right) \beta\left(t_{k}[u]\right)}\right)^{\alpha_{l}[u]} .
\end{aligned}
$$

- If $\beta\left(t_{k}[u]\right)$ is scheduled for two subsequent time-slots, it either transmits or remains quiet in both,

$$
\begin{aligned}
& \operatorname{Pr}\left[\alpha_{k}[u]=\alpha_{\Lambda(k)}[u] \mid \Lambda(k)=k-1, k \neq 0\right]=1, \\
& \operatorname{Pr}\left[\alpha_{k}[u] \neq \alpha_{\Lambda(k)}[u] \mid \Lambda(k)=k-1, k \neq 0\right]=0 .
\end{aligned}
$$

Equations (2) to (7) can be substituted by a simplified expression as follows:

$$
\begin{aligned}
& \operatorname{Pr}\left[\alpha_{k}[u] \mid\left\langle\alpha_{l}[u]\right\rangle_{1 \leq l \leq k-1}\right] \\
& \mid \prod_{l=\Lambda(k)+1}^{k-1}\left(p_{\beta\left(t_{l}[u]\right) \beta\left(t_{k}[u]\right)}\right)^{\alpha_{l}[u]\left(1-\alpha_{\Lambda(k)}[u]\right)} \\
& \\
&-\left|\alpha_{k}[u]-\alpha_{\Lambda(k)}[u]\right|
\end{aligned}
$$

Therefore, $\mathbf{a}_{:, u}\left(t_{u}\left[T_{u}\right]\right)=\mathbf{1}_{N, 1}$, i.e., the event that all nodes receive at least one correct copy of the packet $\mu[u]$ after $T_{u}$ 


\begin{tabular}{|c|c|c|c|c|c|c|}
\hline & $\mid \mu[1|\mu[2]| \mu[3]|\mu[4]|$ & $|\mu[1]| \mu[2]|\mu[3]| \mu[4] \mid$ & $|\mu[1]| \mu[2]|\mu[3]| \mu[4] \mid$ & $|\mu[1]| \mu[2]|\mu[3]| \mu[4] \mid$ & $|\mu[1]| \mu[2]|\mu[3]| \mu[4] \mid$ & $|\mu[1]| \mu[2]|\mu[3]| \mu[4] \mid$ \\
\hline $\mathrm{S}_{1}$ & $\triangle$ & & & & & \\
\hline $\mathrm{S}_{2}$ & $\theta$ & & & & & \\
\hline $\mathrm{S}_{3}$ & & & & & & \\
\hline $\mathrm{S}_{4}$ & $\theta$ & & & $\varnothing$ & & \\
\hline & $t=1$ & $t=2$ & $t=3$ & $t=4$ & $t=5$ & $t=6$ \\
\hline & $\mathbf{A}(1)=\left[\begin{array}{llll}1 & 0 & 0 & 0 \\
0 & 0 & 0 & 0 \\
1 & 0 & 0 & 0 \\
0 & 0 & 0 & 0\end{array}\right]$ & $\mathbf{A}(2)=\left[\begin{array}{llll}1 & 0 & 0 & 0 \\
0 & 1 & 0 & 0 \\
1 & 0 & 0 & 0 \\
0 & 1 & 0 & 0\end{array}\right]$ & $\mathbf{A}(3)=\left[\begin{array}{llll}1 & 0 & 1 & 0 \\
0 & 1 & 0 & 0 \\
1 & 0 & 1 & 0 \\
1 & 1 & 1 & 0\end{array}\right]$ & $\mathbf{A}(4)=\left[\begin{array}{llll}1 & 0 & 1 & 0 \\
0 & 1 & 1 & 1 \\
1 & 1 & 1 & 1 \\
1 & 1 & 1 & 1\end{array}\right]$ & $\mathbf{A}(5)=\left[\begin{array}{llll}1 & 1 & 1 & 1 \\
0 & 1 & 1 & 1 \\
1 & 1 & 1 & 1 \\
1 & 1 & 1 & 1\end{array}\right]$ & $\mathbf{A}(6)=\left[\begin{array}{llll}1 & 1 & 1 & 1 \\
1 & 1 & 1 & 1 \\
1 & 1 & 1 & 1 \\
1 & 1 & 1 & 1\end{array}\right]$ \\
\hline & ite trar & $\triangle$ ori & & & & ully decoded packets \\
\hline
\end{tabular}

Fig. 3. An example of time-slot allocation for $N=4, K=2$ and $L=3$. The redundant time-slots 5 and 6 are allocated for node $S_{3}$ and $S_{4}$, respectively.

transmission attempts, has the probability as shown in (9) below.

$$
\begin{aligned}
& \operatorname{Pr}\left\{\mathbf{a}_{:, u}\left(t_{u}\left[T_{u}\right]\right)=\mathbf{1}_{N, 1}\right\}=
\end{aligned}
$$

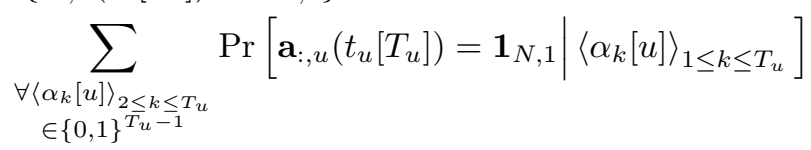

$$
\begin{aligned}
& \times \prod_{k=1}^{T_{u}} \operatorname{Pr}\left[\alpha_{k}[u] \mid\left\langle\alpha_{l}[u]\right\rangle_{1 \leq l \leq k-1}\right] \\
& =\sum_{\substack{\forall\left\langle\alpha_{k}[u]\right\rangle_{2 \leq k \leq T} \\
\in\{0,1\}^{T_{u}-1}}} \prod_{s=1}^{N} \mid 1-\alpha_{\Lambda\left(T_{u}\right)}[u] \\
& -\prod_{h=\Lambda\left(T_{u}\right)+1}^{T_{u}-1}\left(p_{\beta\left(t_{h}[u]\right)}\right)^{\alpha_{h}[u]\left(1-\alpha_{\Lambda\left(T_{u}\right)}[u]\right)} \\
& \times \prod_{j=2}^{T_{u}-1} \prod_{l=\Lambda(j)+1}^{j-1}\left(p_{\beta\left(t_{l}[u]\right) \beta\left(t_{j}[u]\right)}\right)^{\alpha_{l}[u]\left(1-\alpha_{\Lambda(j)}[u]\right)} \\
& -\left|\alpha_{j}[u]-\alpha_{\Lambda(j)}[u]\right| \mid
\end{aligned}
$$

As $a_{s, u}\left(t_{u}\left[T_{u}\right]\right)=a_{s, u}(N+K)$, the probability $B_{p}$ that all nodes successfully receive the broadcast message is:

$$
\begin{aligned}
B_{p} & =\operatorname{Pr}\left\{a_{s, u}(N+K)=1, \forall s, u \in\{1,2, \ldots, N\}\right\} \\
& =\prod_{u=1}^{N} \operatorname{Pr}\left\{\mathbf{a}_{:, u}\left(t_{u}\left[T_{u}\right]\right)=\mathbf{1}_{N, 1}\right\} .
\end{aligned}
$$

Given that the sequence of broadcasters in the first phase of $N$ time-slots is fixed and using (10) as an objective function to maximize, a wide range of combinatoric algorithms can be applied to find solutions for the second phase of $K$ time-slots. In this work, however, two simple algorithms are considered as follows:

Greedy search: makes the locally best choice in each timeslot. Although a greedy algorithm in general is not guaranteed to find the globally optimal solution, it may yield a local optimal one in polynomial time. In this specific problem, using greedy search yields the worstcase complexity of $\left(\begin{array}{c}N \\ L\end{array}\right) N K^{2}$, where $(\cdot)$ represents the binomial coefficient.

Memory-based heuristic: in [11], a low-complexity algorithm is proposed. Although it is more complex than greedy search, it has a much higher chance to find a globally optimal solution, and on average, yields much better results as compared to greedy search.

\section{Numerical EXAMPles}

In this section, numerical results are presented for the performance of the proposed strategy in comparison with that of the analysis. The parameter setting suggested by the empirical results in [12] is used, i.e., $N_{0}=-99 \mathrm{dBm}, \theta_{t h}=11$ $\mathrm{dB}$ for QPSK modulation and code rate $3 / 4$, while $P_{t}=1$ $\mathrm{mW}$. For any pair of nodes $(i, j)$, we assume a channel with Nakagami- $m$ fading, together with additive white Gaussian noise (AWGN). The channel coefficient $g_{i j}$ is characterized by an i.n.i.d Nakagami- $m$ distribution, which has the probability 


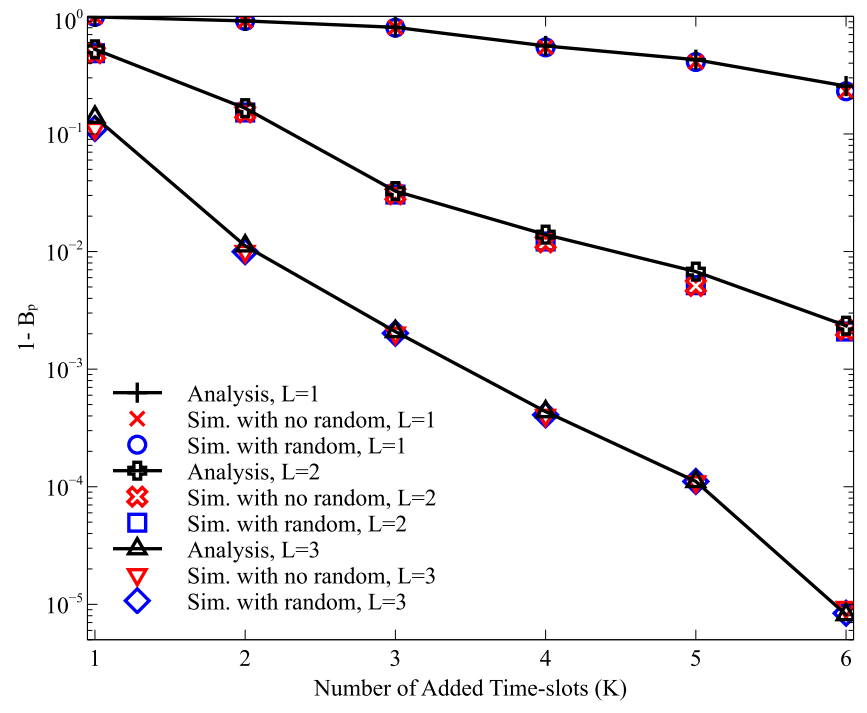

Fig. 4. Verifying the analysis by simulation with various $L$.

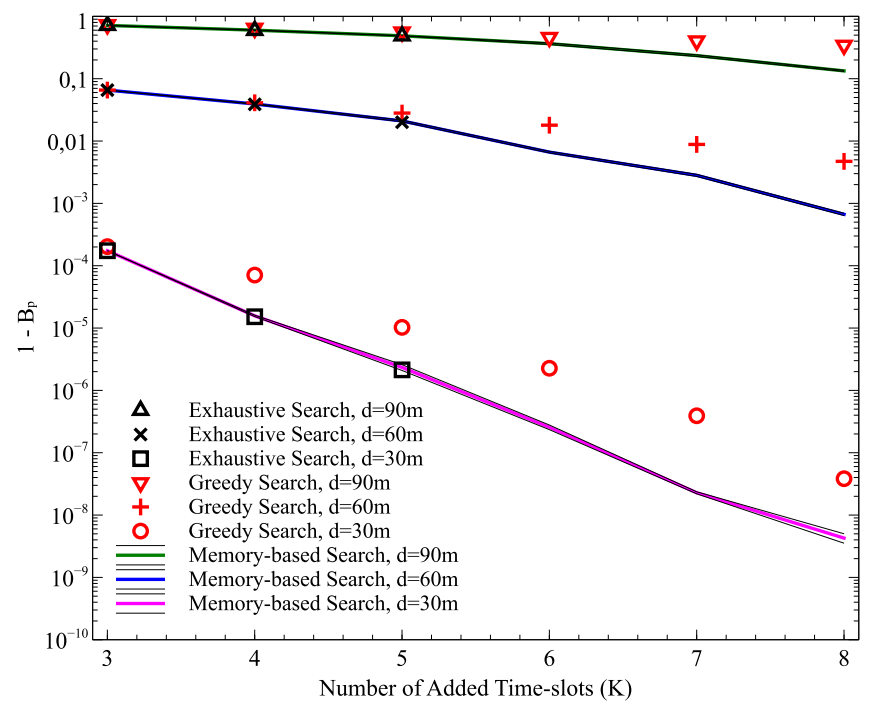

Fig. 5. Comparison of different searching algorithms, $L=2$.

with much lower complexity. Finally, we can see that given eight redundant time-slots, all nodes receive all four packets with probability of error less than $10^{-8}$ although the distance between the end nodes in the line network is 270 meters.

\section{CONCLUSions}

In this paper, we have proposed and evaluated a relaying scheme with packet aggregation that is suitable for half-duplex all-to-all broadcast in time-critical ultra-reliable wireless networks. Moreover, we have derived closed-form analytical expressions for the probability that each node successfully decodes all original packets of the other nodes. Numerical examples and simulations have verified the correctness of the derivation. The results also show that randomly choosing additional packets to aggregate does not help to increase the probability of all-to-all successful decoding. The resulting approach is suitable for wireless distributed control systems, where sensor data from several different nodes must be timely and reliably available at all places where controller decisions are made. It can be concluded that our scheme achieves ultrareliable low-latency communications in wireless networks.

\section{REFERENCES}

$B_{p}$. This suggests that randomly adding packets to aggregate help the nodes to successfully decode all original messages transmitted from all other nodes in the network.

In Fig. 5, we compare the performance of the two proposed algorithms and benchmark them with exhaustive search in a regular line network of four nodes where $L=2$, the path loss exponent $\gamma_{P L}=4$ and $m_{i j}=1, \forall i, j$. Exhaustive search can only be performed for five or fewer time slots, for complexity reasons. The memory-based search algorithm is repeated ten times to provide samples which are $99 \%$ confident. As shown in the figure, the memory-based search algorithm performs very close to the optimal exhaustive search, but at manageable complexity. The greedy algorithm also gives acceptable results
[1] M. Ivanov, F. Brännström, A. G. i Amat, and P. Popovski, "All-to-all broadcast for vehicular networks based on coded slotted aloha," in Proc. IEEE Int. Conf. Commun. Wksp., London, UK, Jun. 2015, pp. 20462050.

[2] W. Liang, R. Brent, Y. Xu, and Q. Wang, "Minimum-energy all-toall multicasting in wireless ad hoc networks," IEEE Trans. Wireless Commun., vol. 8, no. 11, pp. 5490-5499, 2009.

[3] R. Gandhi, Y.-A. Kim, S. Lee, J. Ryu, and P.-J. Wan, "Approximation algorithms for data broadcast in wireless networks," IEEE Trans. Mobile Comput., vol. 11, no. 7, pp. 1237-1248, 2012.

[4] L. Ma, Z. Lin, Z. Zhang, G. Mao, and B. Vucetic, "Reliability of allto-all broadcast with network coding," in Proc. IEEE Global Commun. Conf., Atlanta, GA, Dec. 2013, pp. 1991-1996.

[5] Y.-C. Tseng, S.-Y. Ni, Y.-S. Chen, and J.-P. Sheu, "The broadcast storm problem in a mobile ad hoc network," Wireless Netw., vol. 8, no. 2-3, pp. 153-167, 2002. 
[6] L. Krishnamachari, D. Estrin, and S. Wicker, "The impact of data aggregation in wireless sensor networks," in Proc. Int. Conf. Distributed Comput. Sys. Wksp, Vienna, Austria, Jul. 2002, pp. 575-578.

[7] S. Girs, A. Willig, E. Uhlemann, and M. Bjorkman, "Scheduling for source relaying with packet aggregation in industrial wireless networks," IEEE Trans. Ind. Infomat., vol. 12, no. 5, pp. 1855-1864, 2016.

[8] J. Neander, T. Lennvall, and M. Gidlund, "Prolonging wireless hart network lifetime using packet aggregation," in 2011 IEEE Int. Symp. Ind. Electron., Gdansk, 2011, pp. 1230-1236.

[9] M. K. Simon and M.-S. Alouini, Digital communication over fading channels. John Wiley \& Sons, 2005, vol. 95.

[10] L.-N. Hoang, E. Uhlemann, and M. Jonsson, "A novel relaying scheme to guarantee timeliness and reliability in wireless networks," in Proc. IEEE Global Commun. Conf. Wksp., Washington, DC, Dec. 2016.

[11] _ _ "Low complexity algorithm for efficient relay assignment in unicast/broadcast wireless networks," in Proc. IEEE Veh. Tech. Conf. (accepted for publication), Sydney, Australia, Jun. 2017.

[12] D. Jiang, Q. Chen, and L. Delgrossi, "Optimal data rate selection for vehicle safety communications," in Proc. ACM Int. Wksp. Veh. Internetw. Syst. Applicat., San Francisco, CA, Sep. 2008, pp. 30-38. 